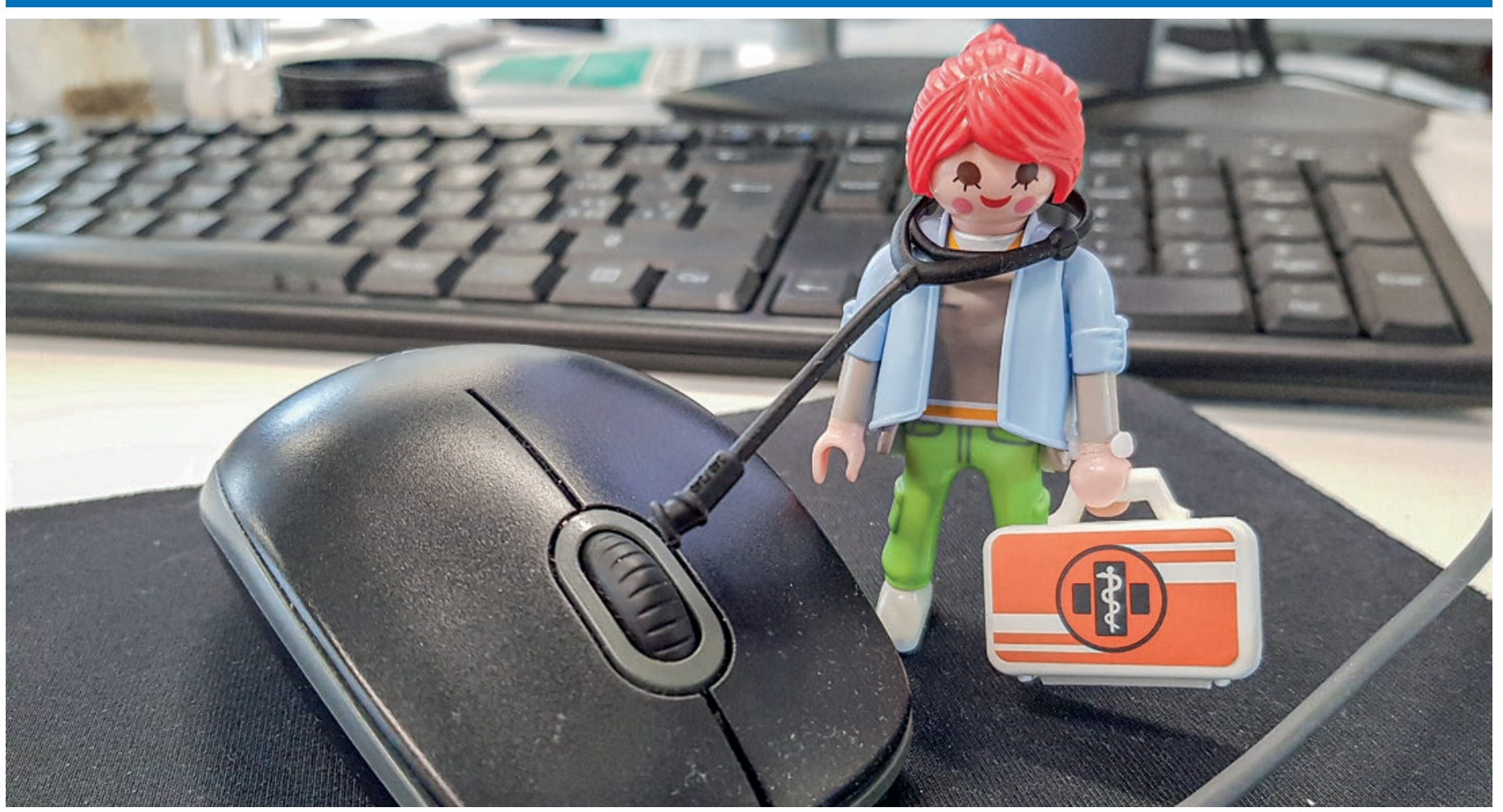

\title{
Effizienz und Patientensicherheit von Klinikinformationssystemen
}

\section{Simone Fischer, ${ }^{a, c}$, David L. B. Schwappach ${ }^{b, c, d}$, Reinhold Sojer ${ }^{e, f}$, Esther Kraft ${ }^{g}$}

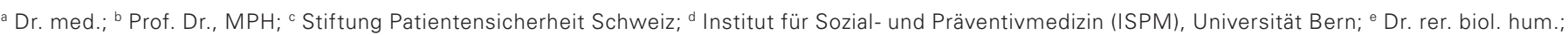
${ }^{\mathrm{f}}$ Leiter Abteilung Digitalisierung/eHealth, $\mathrm{FMH} ;{ }^{\mathrm{g}}$ Leiterin Daten Demographie und Qualität DDQ, FMH

Im Gesundheitswesen können elektronische Informationssysteme die Effizienz und die Sicherheit in der Gesundheitsversorgung verbessern. Unsachgemäss konzipiert, implementiert und angewendet kann dieses Potenzial jedoch nicht ausgeschöpft werden, und es kommt zu Fehlern, die wiederum zur Schädigung von Patientinnen und Patienten sowie zu Ineffizienz und Frustration beim Gesundheitspersonal führen können.

In den letzten Jahrzehnten hat sich die Digitalisierung im Gesundheitswesen massiv beschleunigt und $\mathrm{zu}$ einer mittlerweile weit verbreiteten Einführung und Nutzung von Gesundheitsinformationstechnologien wie beispielsweise Klinikinformationssystemen (KIS) und elektronischen Verschreibungssystemen geführt [1]. KIS sind mittlerweile der zentrale Speicher für Gesundheitsinformationen in Spitälern und die Basis für klinische Entscheidungen sowie elektronische Verordnungen. Gemäss dem Swiss e-Health Barometer 2020 arbeiten nahezu alle Schweizer Spitalärztinnen und -ärzte (91\%) mit einem elektronischen System, und von
83\% werden Medikamente routinemässig elektronisch verordnet [2]. Diese Werte halten sich seit 2017 relativ stabil, was für einen breiten Einsatz solch elektronischer Systeme in den Spitälern spricht.

Diese Technologien können die Effizienz, Sicherheit und Qualität in der Gesundheitsversorgung verbessern. In internationalen Studien wurde beispielsweise von einer Erleichterung bei den Medikamentenverordnungen, weniger unerwünschten Arzneimittelereignissen, einem Rückgang fehlerhafter Doppel-Untersuchungen und geringeren Kosten berichtet [3, 4]. Werden diese Systeme jedoch inadäquat konzipiert, entwickelt, imple- 
mentiert und angewendet, so kann es zu unerwünschten Folgen, wie zusätzlicher Arbeit für Ärztinnen und Ärzten, erschwerten Arbeitsabläufen und neuen Arten von Fehlern, kommen, die wiederum zu Problemen bei der Patientensicherheit führen [5]. Nebst potenziellen Patientenschäden führt die Verwendung komplizierter und unzureichender elektronischer Systeme zu Ineffizienz und Frustration beim Gesundheitspersonal und trägt bei Ärztinnen und Ärzten zum Auftreten von Burnouts bei [3]. Ein weit verbreiteter Faktor, der zu diesen Problemen beiträgt, ist deren schlechte Gebrauchstauglichkeit, insbesondere bezüglich Benutzerfreundlichkeit (Usability). Diese definiert, in welchem Mass Endbenutzer ihre beabsichtigten Aufgaben effizient und zufriedenstellend mit dem System erledigen können. [6]

\section{Mehr Fehler bei schlechtem Design}

Spezifisch für KIS konnte in einer amerikanischen Studie nachgewiesen werden, dass Ärztinnen und Ärzten häufig Fehler bei der Aufgabenerledigung im KIS unterlaufen und dass sich diese Häufigkeit stark zwischen den KIS und deren jeweiliger Implementierung unterscheidet [6]. Eine in der Schweiz durchgeführte prospektive Studie von Pfeiffer et al. [7] untersuchte Patientensicherheitsprobleme von Gesundheitsinformationstechnologien in drei onkologischen Ambulatorien. In den drei Ambulatorien konnte nachgewiesen werden, dass die derzeitige Implementierung und das Design der elektronischen Informationssysteme (z.B. durch die Vielzahl der Quellen und die fragmentierte Ablage) kein adäquates Management von Informationen erlauben und ein Risiko für die Patientensicherheit darstellen.

\section{Nebst potenziellen Patientenschäden führt die Verwendung komplizierter Systeme zu Frustra- tion beim Gesundheitspersonal und trägt zum Auftreten von Burnouts bei.}

Aktuell im Einsatz stehende Schweizer KIS sind häufig technologisch veraltet und bieten wenige Möglichkeiten, neue Technologien einzubinden. 49\% der Spitalärztinnen und -ärzte sind mit dem KIS in ihrem Spital unzufrieden [2]. Nebst Verbesserungspotenzial bei den Benutzeroberflächen und bei den Schnittstellen werden am häufigsten die Funktionalität der Systeme sowie deren Komplexität und Langsamkeit bemängelt [2] . Es ist erstaunlich, dass trotz der inzwischen weit verbreiteten Nutzung von KIS in Schweizer Spitälern wenig über deren Unterschiede und Sicherheit bekannt ist. Es mangelt nicht nur an wissenschaftlichen Studien, es gibt in der Schweiz auch keine Richtlinien oder Standards für Testungen des Systemdesigns im Rahmen der Entwicklung und Implementierung von KIS

\section{Forschungsprojekt zu zwei KIS}

In einer durch die Stiftung Patientensicherheit Schweiz konzipierten und durchgeführten Studie wurde schweizweit erstmalig die Effizienz und Fehleranfälligkeit von zwei in der Schweiz gängigen KIS (Systeme A/B) untersucht. Finanziell wurde das Forschungsprojekt durch die Stiftung Lindenhof Bern, Fonds Lehre und Forschung, die Hanela Stiftung sowie die FMH unterstützt. Mittels eines klassischen szenariobasierten Usability-Tests [8] unter experimentellen Bedingungen erledigten Ärztinnen und Ärzte aus dem Fachbereich der Inneren Medizin in vier verschiedenen Spitälern (Spital 1A, 2A, 3B, 4B) typische Routineaufgaben. Diese waren in sechs klinische Szenarien, also simulierten Patientenfällen, integriert und umfassten das elektronische Verordnen von Medikamenten, Radiologie- und Laborverordnungen und andere typische Aufgaben wie beispielsweise die Erstellung von Rezepten. Box 1 zeigt Beispiele von zwei Szenarien. Als Parameter für die Effizienz wurden zwischen den Spitälern die Anzahl der Mausklicks analysiert und die Zeit, die zur Erledigung der Aufgabe benötigt wurde. Zur Untersuchung der Patientensicherheit wurden die unterschiedlichen Fehlertypen, Fehleranzahl und -rate sowie die Genauigkeit zwischen den Spitälern evaluiert.

Insgesamt beteiligten sich 58 Ärztinnen und 42 Ärzte $(\mathrm{N}=100)$ aus vier verschiedenen Spitälern an der Studie. Davon waren 70\% in der Funktion als Assistenzund Oberärztinnen und -ärzte sowie $30 \%$ als leitende und Chefärztinnen und -ärzte tätig. Zwischen den Spitälern gab es hinsichtlich der Probandencharakteristika keine signifikanten Unterschiede. Fast alle Ärztinnen und Ärzte (88\%) hatten zuvor in irgendeiner Form eine KIS-Schulung erhalten.

\section{Effizienz und Fehleranfälligkeit}

Im Rahmen des Forschungsprojektes konnten grosse Unterschiede in der Effizienz und Fehleranfälligkeit zwischen den Spitälern und den KIS gezeigt werden. Zur Erledigung aller Aufgaben im Szenario 3 (siehe Box 1) benötigten die Ärztinnen und Ärzte durchschnittlich 87 Mausklicks in 5:25 Minuten. Mausklicks und Zeit variierten jedoch stark zwischen den Ärztinnen und Ärzten, von Minimum (Min) 32 bis Maximum (Max) 192 Klicks und 1:45 bis 11:52 Minuten. Im gesamten Szenario 3 wur- 


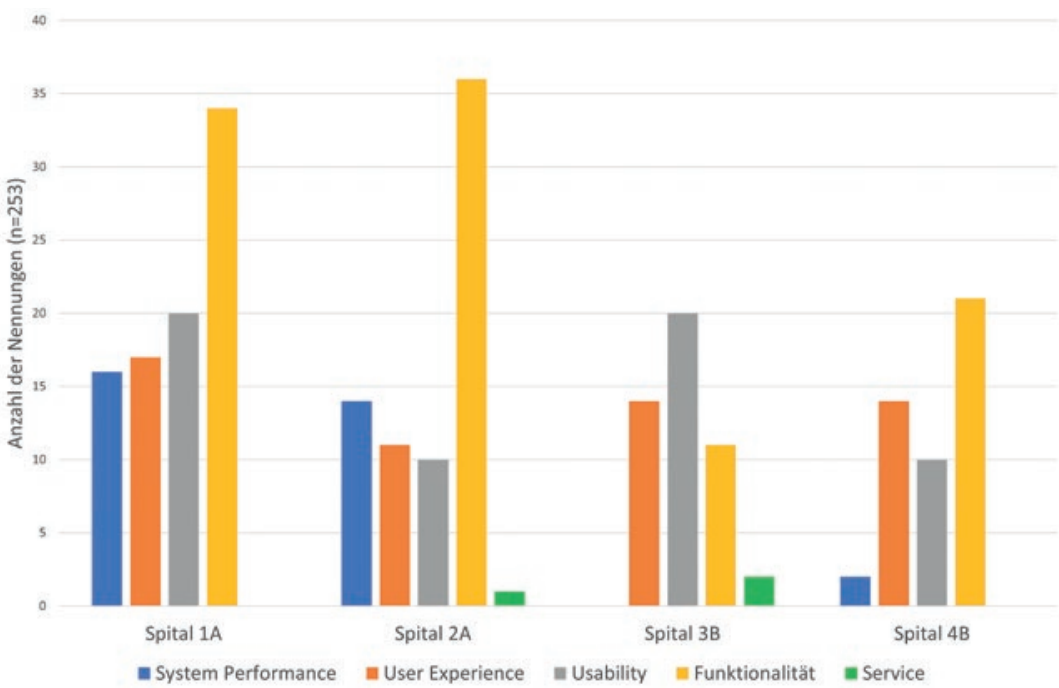

Abbildung 1: Verbesserungspotenzial beim KIS nach Spital. Auswertung der mündlichen Auskünfte der teilnehmenden Ärztinnen und Ärzte. den im Mittel 2,3 Fehler (Max 11 Fehler) gemacht, was einer Fehlerrate von 20,5\% entspricht. Von den 100 Studienteilnehmerinnen und -teilnehmern erledigten lediglich 27\% die Aufgabe vollständig korrekt.

Insbesondere die im Szenario 5 integrierte komplexere Aufgabe zum Ausschleichen von Prednison (siehe Box 1) zeigte grosse Mängel bei den Systemen. Allein für die Durchführung dieser einzigen Aufgabe benötigten die Ärztinnen und Ärzte im Durchschnitt knapp 6 Minuten mit einer durchschnittlichen Anzahl von 85 Mausklicks, 65\% machten 1 bis 4 Fehler. Auch hier zeigten sich zwischen den Spitälern grosse Unterschiede. In Tabelle 1 sind die detaillierten Ergebnisse der Prednison-Aufgabe nach Spital (Spital 1A-4B) sichtbar. Zusätzlich wurden Ergebnisse einer amerikanischen Studie von Ratwani et al. (blaue Spalten) mit identischem Studiendesign und identischer Aufgaben-

\section{Box 1: Beispiele von Szenarien}

Szenario 3: Thoraxschmerzen

Öffnen Sie bitte den Fall von Herrn Antonio Da Silva, geb. 03.2.1961, oder die Fallnummer XX.

\section{Einleitung}

Der 59-jährige Herr Da Silva klagt überThoraxschmerzen mit Ausstrahlung in den linken Arm, dazu begleitend Dyspnoe. Die Schmerzen fühlen sich ähnlich an wie beim letzten Herzinfarkt. Das initiale EKG zeigt einen normalen Sinusrhythmus mit unspezifischen ST-Wellen-Anomalien.

Erste Massnahmen

Erteilen Sie folgenden Laborauftrag:

- Kleines Blutbild

- Chemie: NA, K, Kreatinin

- Troponin

Bitte verordnen Sie jetzt!

Ausserdem braucht es die weiteren Verordnungen:

- Röntgen:Thorax in 2 Ebenen Fragestellung: Pneumothorax?

- $\quad$ Einmaldosis Acetylsalicylsäure (Aspegic $\left.{ }^{\circledR}\right) 250$ mg i.v.

Verordnen Sie bitte!

Weitere Massnahmen und Procedere

Sie bekommen von einer Pflegefachperson mitgeteilt, das der $\mathrm{Pa}$ tient einen Sättigungsabfall auf 90 hat. Zudem erhalten Sie in der Zwischenzeit die Laborresultate zurück, das Troponin ist leicht erhöht.

Bitte führen Sie folgende Verordnungen aus:

- $\quad$ Sauerstoff $21 / \mathrm{min}$

- Heparin-Bolus von 5000 IE als i.v. Inj., danach kontinuierlich $30000 \mathrm{IE} / \mathrm{Tag}$

- Troponin-Test soll in 3 Stunden wiederholt werden

Bitte jetzt verordnen!
Szenario 5: COPD

Öffnen Sie bitte den Fall von Frau Susanne Nötzli, geb. 1.5.1968, oder die Fallnummer XX.

\section{Einleitung}

Die 52-jährige Frau Nötzli wurde am Vortag aufgrund einer Exazerbation ihrer bekannten COPD hospitalisiert. Auf der Abteilung kommt es erneut zur akuten Dyspnoe. Bei der körperlichen Untersuchung zeigt sich ein Giemen ubiquitär mit verlängertem Expirium, eine Atemfrequenz von 22 sowie eine Sauerstoffsättigung von $87 \%$ bei Raumluft.

Erste Massnahmen

Bitte verordnen Sie folgende Medikamente:

- Salbutamol und Ipratropiumbromid (Dospir ${ }^{\circledR}$, Ipramol $^{\circledR}$ ), Inhalation sofort, anschliessend $4 x$ tgl als Fixmedikation

- Methylprednisolon (Solu-Medrol ${ }^{\circledR}$ ) 40 mg i.v., Einmaldosis

Verordnen Sie bitte!

Weitere Massnahmen und Procedere

Es kommt zu einer Regression der Symptomatik.

Verordnen Sie für den Folgetag:

- Prednison oder Prednisolon (Spiricort ${ }^{\circledR}$ ) 60 mg mit einer Reduktion um $10 \mathrm{mg}$ alle 2 Tage für insgesamt 12 Tage

Bitte jetzt verordnen!

Sie überprüfen die weitere Medikation der Patientin und intensivieren die therapeutischen Massnahmen.

Veranlassen Sie folgende Verordnungen

- Ultibro ${ }^{\circledR}$ Breezhaler $^{\circledR} 110 \mathrm{mcg} / 50 \mathrm{mcg} 1 \times \mathrm{tgl}$

- Physiotherapierezept für Atemtherapie

- Flaschenblasen

Verordnen Sie jetzt! 


\begin{tabular}{|c|c|c|c|c|c|c|c|c|}
\hline $\begin{array}{l}\text { Spital (1-8) } \\
\text { und } \\
\text { KIS (A-D) } \\
\text { Mean (SD) }\end{array}$ & $\begin{array}{l}\text { Spital 1A } \\
\mathrm{n}=25\end{array}$ & $\begin{array}{l}\text { Spital 2A } \\
n=26\end{array}$ & $\begin{array}{l}\text { Spital 3A } \\
n=24\end{array}$ & $\begin{array}{l}\text { Spital 4B } \\
n=25\end{array}$ & $\begin{array}{l}\text { Spital } \\
5 C^{*} \\
n=14\end{array}$ & $\begin{array}{l}\text { Spital } \\
6 C^{*} \\
n=15\end{array}$ & $\begin{array}{l}\text { Spital } \\
7 D^{*} \\
n=14\end{array}$ & $\begin{array}{l}\text { Spital } \\
8 D^{*} \\
n=12\end{array}$ \\
\hline Mausklicks (n) & $101.9(27.2)$ & $87.2(48.1)$ & $71.6(32.5)$ & $77.6(38.6)$ & $32.2(16.6)$ & $20.0(32.8)$ & $42.3(17.6)$ & $28.2(5.7)$ \\
\hline $\begin{array}{l}\text { Time-on-task } \\
\text { (Sek) }\end{array}$ & $\begin{array}{l}391.3 \\
(124.2)\end{array}$ & $\begin{array}{l}353.9 \\
(172.9)\end{array}$ & $\begin{array}{l}266.8 \\
(160.8)\end{array}$ & $\begin{array}{l}300.5 \\
(168.3)\end{array}$ & $\begin{array}{l}148.6 \\
(76.1)\end{array}$ & $\begin{array}{l}152.7 \\
(163.4)\end{array}$ & $\begin{array}{l}175.1 \\
(73.0)\end{array}$ & $\begin{array}{l}178.7 \\
(62.6)\end{array}$ \\
\hline Fehlerrate ${ }^{\&}$ & $142 \%$ & $178 \%$ & $114 \%$ & $133 \%$ & $16.7 \%$ & $41.7 \%$ & $50 \%$ & $40 \%$ \\
\hline $\begin{array}{l}\text { Häufigster } \\
\text { Fehlertyp }\end{array}$ & $\begin{array}{l}\text { Falsche Dos } \\
\text { Stoppdatum }\end{array}$ & ung 4 & es Start & & Fal & erung & & \\
\hline
\end{tabular}

Mean (SD) - Mittelwert (Standardabweichung); $n$ - Anzahl Mausklicks/Probanden pro Spital;

Sek - Sekunden; * - Ergebnisse von Ratwani et al. (USA) [6];

\& Fehlerraten $>100 \%$ sind möglich, wenn mehrere Fehler pro Aufgabe gemacht wurden.

Tabelle 2: Ergebnisparameter nach Art der Aufgabe über alle Szenarien hinweg.

\begin{tabular}{|c|c|c|c|c|c|c|c|c|}
\hline \multirow[t]{2}{*}{ Aufgabenart } & \multicolumn{2}{|c|}{$\begin{array}{l}\text { Medikamenten- } \\
\text { verordnungen } \\
\text { (17 Aufgaben) }\end{array}$} & \multicolumn{2}{|c|}{$\begin{array}{l}\text { Laborverordnungen } \\
\text { (7 Aufgaben) }\end{array}$} & \multicolumn{2}{|c|}{$\begin{array}{l}\text { Radiologieverordnungen } \\
\text { (6 Aufgaben) }\end{array}$} & \multicolumn{2}{|c|}{$\begin{array}{l}\text { Sonstige Verordnungen } \\
\text { (8 Aufgaben) }\end{array}$} \\
\hline & Mean (SD) & Min/Max & Mean (SD) & Min/Max & Mean (SD) & Min/Max & Mean (SD) & Min/Max \\
\hline Mausklicks (n) & $276.3(72.2)$ & $146 / 480$ & $33.3(10.9)$ & $15 / 78$ & $62.8(20.7)$ & $32 / 142$ & $104.9(33.7)$ & $55 / 255$ \\
\hline Time-on-task (Sek) & $\begin{array}{c}1037.0 \\
(398.6) \\
\end{array}$ & $400 / 2046$ & $106.6(50.2)$ & $41 / 298$ & $281.2(197.4)$ & $96 / 1780$ & $462.8(492.8)$ & $140 / 3373$ \\
\hline Fehlerrate (\%) & $32.0(22.7)$ & $0 / 135.3$ & $14.6(31.1)$ & $0 / 129$ & $47.5(30.0)$ & $0 / 183$ & $41.9(21.7)$ & $0 / 87.5$ \\
\hline Häufigster Fehlertyp & Falsche Dos & ierung & Falscher Zei & tpunkt & Falsche Eben & & $\begin{array}{l}\text { Unvollständi } \\
\text { Verordnung }\end{array}$ & \\
\hline
\end{tabular}

Mean (SD) - Mittelwert (Standardabweichung); Min/Max - Minimum/Maximum; n - Anzahl Mausklicks; Sek - Sekunden.

stellung integriert [6]. Dort wurden zwei in den USA marktführende KIS in ebenfalls vier Spitälern untersucht (Spital 5C-8D). Im Vergleich zu den amerikanischen Spitälern benötigten die Ärztinnen und Ärzte in der Schweiz meist doppelt bis dreifach so viel Zeit und Klicks und machten um ein Vielfaches mehr Fehler. Bei den in der Schweiz genutzten KIS war System B dem System A überlegen.

Eine Betrachtung der Schweizer Ergebnisparameter nach Aufgabenart, also Medikamenten-, Labor-, Radiologie- oder sonstiger Verordnung, zeigt ebenfalls eine sehr grosse Spannweite (Min/Max) der Werte. So entspricht beispielsweise die maximal benötigte Zeit zur Erledigung aller Radiologieverordnungen einem 18-Fachen der schnellsten Zeit (siehe Tab. 2). Auch bei den Fehlerhäufigkeiten und -raten sind grosse Spannweiten und generell eine hohe Anzahl sichtbar.

Insgesamt betrachtet benötigten Ärztinnen und Ärzte, die mit System B arbeiteten, zur Erledigung der Aufgaben weniger Mausklicks und Zeit und machten weniger Fehler. Aber auch innerhalb der Spitäler mit dem gleichen System zeigten sich teilweise signifikante Unterschiede. Keiner der 100 Ärztinnen und Ärzte konnte alle 38 Aufgaben ohne einen Fehler abschliessen.

\section{Grössere Zufriedenheit mit System B}

In der Studie konnte zudem nachgewiesen werden, dass die Ärztinnen und Ärzte, die mit dem weniger fehleranfälligen und ressourcenschonenderen System $\mathrm{B}$ arbeiten, mit ihrem System auch signifikant zufriedener waren als die Nutzer von System A. Verbesserungspotenzial beim KIS sahen die Nutzerinnen und Nutzer von System A primär bei der Funktionalität und System-Performance, was von den Ärztinnen und Ärzten mit System B deutlich seltener genannt wurde (siehe Abb. 1). So wurden beispielsweise bei der Funktionalität das Berichtewesen, eine fehlende automatische Übernahme von bereits erfassten Angaben oder die Tatsache, dass nicht mehrere Module parallel geöffnet werden können, bemängelt. Zudem wurde von vielen Systemunterbrüchen und -abstürzen berichtet. Nutzerinnen und Nutzer beider Systeme beklagten generell eine hohe Klicklast sowie Mängel bei der Übersichtlichkeit.

\section{Ressourcenbedarf und Sicherheitsrisiken}

Die Studienergebnisse zeigen eindrücklich, dass bei den untersuchten KIS ein hoher Ressourcenbedarf und durch ihre hohe Fehleranfälligkeit ein Risiko für die 
Patientensicherheit besteht. Die Unterschiede zwischen den Systemen und Spitälern lassen sich primär durch Unterschiede in der Systemfunktionalität und dem -design sowie bei der Implementierung des KIS erklären, d.h. durch lokale Konfigurationen und kundenspezifische Anpassungen sowie durch unterschiedliche Schulung und Unterstützung der Ärztinnen und Ärzte während und nach der Implementierung. Die Differenzen bei den Radiologie-, Labor- und sonstigen Verordnungen verdeutlichen zudem den Einfluss von System-System-Schnittstellen (z.B. KIS und Radiologieinformationssystem [RIS]/Laborinformationssystem [LIS]) auf die Effizienz und Sicherheit, da die Schnittstellen von KIS und den verknüpften RIS/LIS zwischen allen Spitälern unterschiedlich waren. Komplexe und umständlich gestaltete Schnittstellen verursachen viele zusätzliche Klicks und führen zu deutlich mehr Fehlern. Auch die Verbindung der höheren Fehlerraten mit einer längeren Zeit für die Aufgabenerledigung und einer grösseren Anzahl von Mausklicks sind häufig auf eine schlechte Benutzerfreundlichkeit zurückzuführen, die wiederum zu ineffizienten Arbeitsabläufen oder Umgehungen und erhöhter kognitiver Belastung für Ärztinnen und Ärzte führt [9].

\section{Dringender Handlungsbedarf}

Erstmalig für die Schweiz konnten Daten zur Fehleranfälligkeit und Effizienz von gängigen KIS systematisch und unter kontrollierten Bedingungen erhoben werden. Die Resultate sind besorgniserregend. Die Tatsache, dass von der Problematik ungefähr 280 Spitäler mit etwa 170000 Mitarbeiterinnen und Mitarbeitern und jährlich rund 1,5 Millionen stationären Patientinnen und Patienten betroffen sind [10], sollte Spitäler, KIS-Anbieter und politische Entscheidungsträger dazu veranlassen, geeignete Massnahmen und Anforderungen für sicherere Systemdesigns sowie deren Implementierung zu entwickeln. Natürlich erreichen nicht alle Fehler, die im Rahmen der Arbeit mit dem KIS auftreten, auch Patientinnen und Patienten. Die weit überwiegende Mehrzahl wird vorher abgefangen, z.B. durch Rückfragen von Pflegefachpersonen bei unklaren oder inkorrekten Verordnungen. Allerdings führen genau diese Rückfragen und Korrekturen zu den ohnehin häufigen Unterbrechungen und Störungen im Arbeitsablauf von Mitarbeiterinnen und Mitarbeitern im Spital. Diese wiederum können zu Risiken bei den unterbrochenen Arbeiten und zu einer kognitiven Mehrbelastung führen. Auch wenn nicht alle Fehler im KIS direkt zu Patientenschäden führen, so kann doch aus den Daten geschlussfolgert werden, dass die der- zeitigen KIS zu einer Erhöhung der Komplexität und einem schlechten Management von Patientensicherheitsrisiken und zu einer deutlichen Mehrbelastung beim Gesundheitspersonal führen können.

\section{Fazit}

Die Studie zu Effizienz und Patientensicherheit von KIS beleuchtet einen in der Schweiz noch weitgehend unerforschten Bereich und liefert erste relevante Erkenntnisse zu Schweizer KIS. Die Ergebnisse legen nahe, dass die in der Schweiz breit etablierten Systeme fehleranfällig und ineffizient sind und damit die $\mathrm{Pa}$ tientensicherheit sowie die Gesundheit der Ärztinnen und Ärzte systematisch gefährden können. Unter Berücksichtigung, welch schwerwiegenden Folgen inadäquat konzipierte, entwickelte, implementierte und angewendete Systeme für eine Vielzahl von Betroffenen haben können, besteht ein dringender Handlungsbedarf im Bereich der Forschung und Regulation von KIS sowie deren Ausgestaltung. Hierfür müssen alle Stakeholder zusammenarbeiten und sich der Problematik ernsthaft und systematisch widmen.

Literatur

1 Classen DC, Holmgren AJ, Co Z, Newmark LP, Seger D, Danforth M, Bates DW. National Trends in the Safety Performance of Electronic Health Record Systems From 2009 to 2018. JAMA Netw Open. 2020;3:e205547. https://doi.org/10.1001/jamanetworkopen.2020.5547

2 gfs.bern. SWISS eHEALTH BAROMETER 2020, 2020. https://e-healthforum.ch/studienergebnisse-2020/ (accessed April 15, 2021).

3 Kataria S, Ravindran V. Electronic health records: A critical appraisal of strengths and limitations, J R Coll Physicians Edinb. 2020;50:2628. https://doi.org/10.4997/JRCPE.2020.309

4 Nuckols TK, Smith-Spangler C, Morton SC, Asch SM, Patel VM, Ander son LJ, Deichsel EL, Shekelle PG. The effectiveness of computerized order entry at reducing preventable adverse drug events and medication errors in hospital settings: A systematic review and meta-analysis. Syst Rev. 2014;3:1-12. https://doi.org/10.1186/2046-4053-3-56

5 Subbe CP, Tellier G, Barach P. Impact of electronic health records on predefined safety outcomes in patients admitted to hospital: A scoping review. BMJ Open. 2021;11:1-11. https://doi.org/10.1136/bmjopen-2020-047446

6 Ratwani RM, Savage E, Will A, Arnold R, Khairat S, Miller K, Fairbanks RJ, Hodgkins M, Hettinger AZ. A usability and safety analysis of electronic health records: A multi-center study. J Am Med Informatics Assoc. 2018;25:1197-201. https://doi.org/10.1093/jamia/ocy088

7 Pfeiffer Y, Zimmermann C, Schwappach DLB. Patient Safety Threats in Information Management Using Health Information Technology in Ambulatory Cancer Care. J Patient Saf. Publish Ah (2020). https:// doi.org/10.1097/PTS.0000000000000640

8 Friedli S. Empirische Untersuchung zweier Varianten des UsabilityTests: Klassischer Usability-Test und Usability-Walkthrough, Fachhochschule Nordwestschweiz, Hochschule für Angewandte Psychologie, 2014. https://irf.fhnw.ch/bitstream/handle/11654/4910/Friedli Stefanie.pdf?sequence=1 (accessed April 30, 2020).

9 Guo U, Chen L, Mehta PH, Electronic health record innovations: Helping physicians - One less click at a time, Heal Inf Manag J. 2007;46:140-4. https://doi.org/10.1177/1833358316689481

10 Bundesamt für Gesundheit (BAG). Statistiken zur Krankenversicherung, 2021. https://spitalstatistik.bagapps.ch/data/download/kzp19 publication.pdf?v=1616491353 (accessed June 9, 2021). 